\title{
Stepanov-Like Asymptotical Almost Periodic Functions and an Application
}

\author{
Yongkun Li 1 , Yaolu Wang, and Jianglian Xiang \\ Department of Mathematics, Yunnan University, Kunming, Yunnan 650091, China \\ Correspondence should be addressed to Yongkun Li; yklie@ynu.edu.cn
}

Received 20 September 2018; Revised 12 November 2018; Accepted 21 November 2018; Published 4 December 2018

Academic Editor: Richard I. Avery

Copyright (C) 2018 Yongkun Li et al. This is an open access article distributed under the Creative Commons Attribution License, which permits unrestricted use, distribution, and reproduction in any medium, provided the original work is properly cited.

\begin{abstract}
In this paper, we first study some basic properties of Stepanov-like asymptotical almost periodic functions including the completeness of the space of Stepanov-like asymptotical almost periodic functions. Then, as an application, based on these and the contraction mapping principle, we obtain sufficient conditions for the existence and uniqueness of Stepanov-like asymptotical almost periodic solutions for a class of semilinear delay differential equations.
\end{abstract}

\section{Introduction}

Almost periodic functions, which are an important generalization of periodic functions, were introduced into the field of mathematics by Bohr $[1,2]$. From the very beginning, the concept of almost periodic function has attracted extensive attention of mathematicians and has led to various extensions and variations of this concept. For example, Stepanov proposed a weaker concept of almost periodic functions in the sense of Bohr. For more details about Stepanov's almost periodic functions, see [3-11].

On the one hand, due to the fact that almost periodic phenomena exist in the real world, more and more scholars are interested in the almost periodicity and its various generalizations. For example, Diagana [12] introduced Stepanovlike pseudo almost periodicity in 2007. The Stepanov-like pseudo almost periodicity is a generalization of the classical pseudo almost periodicity [13]. The concept of Stepanovlike weighted pseudo almost periodicity was introduced by Diagana et al. [14]. This notion is more extensive than Stepanov-like pseudo almost periodicity. Moreover, Diagana also introduced Stepanov-like almost automorphic functions which are a generalization of the classical almost automorphic functions; for more details, see [15]. In 2009, Diagana introduced the notion of Stepanov-like pseudo almost automorphy which generalizes the concept of pseudo almost automorphy [16].
On the other hand, the concept of the asymptotically almost periodicity was introduced into the research field by French mathematician Frechet $[17,18]$. Such a notion is a natural generalization of the concept of the almost periodicity in the sense of Bohr. Since then, asymptotically almost periodic functions have become a very important function class and to find asymptotically almost periodic solutions for differential equations has been a hot topic for researchers. For the basic properties of asymptotical almost periodic functions, we refer the reader to [19] and for some recent papers about the existence of asymptotically almost periodic solutions for differential equations arising in theory and application, we refer the reader to [20-25]. However, up to now, few studies have been done on Stepanov-like asymptotical almost periodic functions [26], but these studies are necessary.

Motivated by the above discussions, in this paper, we first study some basic properties of Stepanov-like asymptotical almost periodic functions. Then, based on these properties and by using the contraction mapping principle, we investigate the existence and uniqueness of Stepanovlike asymptotical almost periodic solutions for a class of semilinear delay differential equations.

\section{Preliminaries}

In this section, we recall some basis definitions and lemmas of Bohr almost periodic functions and Stepanov's 
almost periodic functions which are used throughout this paper.

Let $\left(X,\|\cdot\|_{X}\right)$ be a Banach space and $B C(R, X)$ be the collection of bounded continuous functions from $R$ to $X$ with the norm $\|x\|=\sup _{t \in R}\|x(t)\|_{X}$.

Definition 1 (see [27]). A function $f \in B C(R, X)$ is said to be almost periodic in Bohr sense if for each $\varepsilon>0$ there exists $l=l(\varepsilon)>0$ such that in every interval of length $l$ of $R$ one can find a number $\tau \in(a, a+l)$ with the property

$$
\|f(t+\tau)-f(t)\|_{X}<\varepsilon, \quad t \in R .
$$

We denote the space of all such functions by $A P(R, X)$; the norm of the space is

$$
|f|_{A P}=\sup _{t \in R}\|f(t)\|_{X}
$$

Definition 2 (see [28]). Let $\Lambda$ be a set of some almost periodic functions in Bohr sense. Then $\Lambda$ is uniformly almost periodic family if it is uniformly bounded, equicontinuous and for every $\varepsilon>0$ there exists a number $l>0$ such that every interval of length $l$ contains a number $\tau$ such that

$$
\|f(t+\tau)-f(t)\|_{X}<\varepsilon, \quad \forall t \in R, \forall f \in \Lambda .
$$

Lemma 3 (see [27]). Function $f \in A P(R, X)$ is equivalent to the property of relative compactness for the family $F=\{f(t+$ $h) ; h \in R\}$.

Definition 4 (see [27]). The space $M$ is defined as follows:

$$
M(R, R)=\left\{x: R \longrightarrow R, \sup _{t \in R} \int_{t}^{t+1}|x(s)| d s<+\infty\right\}
$$

with the norm defined by

$$
|x|_{M}=\sup _{t \in R} \int_{t}^{t+1}|x(s)| d s .
$$

Lemma 5 (see [27]). The space $M(R, R)$ is a Banach space.

Definition 6 (see [27]). A function $f \in M(R, R)$ is said to be Stepanov's almost periodic if for every $\varepsilon>0$ there exists $l=l(\varepsilon)>0$ such that each interval $(a, a+l) \subset R$ contains a point $\tau$ with the property

$$
\sup _{t \in R} \int_{t}^{t+1}|f(s+\tau)-f(s)| d s<\varepsilon .
$$

We denote the space of all such functions by $S(R, R)$ and the norm of $S(R, R)$ is

$$
\|f\|_{S}=\sup _{t \in R} \int_{t}^{t+1}|f(s)| d s .
$$

Lemma 7 (see [27]). The space $S(R, R)$ is a Banach space.

Lemma 8 (see [27]). A function $f \in S(R, R)$ if and only if $\varphi(\cdot, \cdot) \in A P(R, L([0,1], R))$, where

$$
\varphi(t, x)=f(t+x), \quad t \in R, x \in[0,1] \subset R .
$$

Lemma 9 (see [27]). A function $f \in S(R, R)$ if and only if $\mathscr{F}=\{f(t+h) ; h \in R\} \subset S(R, R)$ is relatively compact in $S(R, R)$.

Lemma 10 (see [29]). Let $f_{1}, f_{2}, \ldots, f_{n}$ be almost periodic functions in Bohr sense from $R$ into Banach space $X_{1}, X_{2}, \ldots$, $X_{n}$, respectively. Then for each $\varepsilon>0$, all the functions $f_{1}, f_{2}, \ldots$, $f_{n}$ have a common set of $\varepsilon$-almost periods.

\section{Stepanov-Like Asymptotic Almost Periodic Functions and Their Basic Properties}

Let

$$
\begin{aligned}
M_{0} & (R, R) \\
& =\left\{h \in M(R, R)\left|\lim _{t \rightarrow+\infty} \int_{t}^{t+1}\right| h(s) \mid d s=0\right\},
\end{aligned}
$$

then we give the following definition.

Definition 11 (see [26]). A function $f \in M(R, R)$ is said to be a Stepanov-like asymptotical almost periodic function if it can be expressed as

$$
f(t)=g(t)+h(t),
$$

where $g \in S(R, R), h \in M_{0}(R, R)$. The collection of all such functions will be denoted by $A S(R, R)$.

Remark 12. Several equivalent statements of Definition 11 are given by Theorem 1.6.2 in [26].

Remark 13. Obviously, if $f_{1}, f_{2} \in A S(R, R)$ and $\lambda \in R$, then $f_{1}+f_{2}, \lambda f_{1} \in A S(R, R)$.

Lemma 14. The space of $M_{0}(R, R)$ is a Banach space endowed with the norm

$$
\|f\|_{S}=\sup _{t \in R} \int_{t}^{t+1}|f(s)| d s .
$$

Proof. Let $\left\{h_{n}\right\} \subset M_{0}(R, R) \subset M(R, R)$ be a Cauchy sequence, then we can find a function $h \in M(R, R)$ such that

$$
\lim _{n \rightarrow \infty} \sup _{t \in R} \int_{t}^{t+1}\left|h_{n}(s)-h(s)\right| d s=0 .
$$

Hence

$$
\begin{aligned}
\lim _{t \rightarrow+\infty} \int_{t}^{t+1}|h(s)| d s \leq & \lim _{t \rightarrow+\infty} \int_{t}^{t+1}\left|h(s)-h_{n}(s)\right| d s \\
& +\lim _{t \rightarrow+\infty} \int_{t}^{t+1}\left|h_{n}(s)\right| d s \\
\leq & \left\|h_{n}-h\right\|_{S}, \quad \forall n \in N .
\end{aligned}
$$

Since $\lim _{n \rightarrow \infty}\left\|h_{n}-h\right\|_{S}=0$, we deduce that

$$
\lim _{t \longrightarrow+\infty} \int_{t}^{t+1}|h(s)| d s=0
$$

that is, $h \in M_{0}(R, R)$. Thus, $M_{0}(R, R)$ is a Banach space. The proof is complete. 
Lemma 15. Let $f=g+h \in A S(R, R), g \in S(R, R), h \in$ $M_{0}(R, R)$ and $g(t+x) \in A P(R, L([0,1], R)), h(t+x): R \longrightarrow$ $L([0,1], R), f(t+x): R \longrightarrow L([0,1], R), x \in[0,1]$, then

$$
\{g(t+x), t \in R\} \subset \overline{\{f(t+x), t \in R\}}
$$

(the closure of the range of $f$ ).

Proof. Assume that (15) does not hold, then there exist $t_{0} \in R$ and $\varepsilon>0$ such that

$$
\begin{aligned}
& \left\|f(t+x)-g\left(t_{0}+x\right)\right\|_{L([0,1], R)} \\
& \quad=\int_{0}^{1}\left|f(t+x)-g\left(t_{0}+x\right)\right| d x>3 \varepsilon \quad \forall t \in R .
\end{aligned}
$$

Since $g(t+x) \in A P(R, L([0,1], R))$, there exists $l>0$ and for every $n \in Z$, there exists $\tau_{n} \in\left[n l-t_{0}, n l-t_{0}+l\right]$ such that

$$
\begin{aligned}
\| g & \left(t_{0}+x+\tau_{n}\right)-g\left(t_{0}+x\right) \|_{L([0,1], R)} \\
& =\int_{0}^{1}\left|g\left(t_{0}+x+\tau_{n}\right)-g\left(t_{0}+x\right)\right| d x<\varepsilon .
\end{aligned}
$$

By using the uniform continuity on $R$ of the almost periodic function $g(t+x)$, there exists $K_{0} \in N$ such that $K_{0} \geq 2$ and for all $t \in\left[t_{0}+\tau_{n}-l / K_{0}, t_{0}+\tau_{n}+l / K_{0}\right]$,

$$
\begin{aligned}
& \left\|g(t+x)-g\left(t_{0}+x+\tau_{n}\right)\right\|_{L([0,1], R)} \\
& \quad=\int_{0}^{1}\left|g(t+x)-g\left(t_{0}+x+\tau_{n}\right)\right| d x<\varepsilon .
\end{aligned}
$$

From (16)-(18), it follows that

$$
\begin{aligned}
& \int_{0}^{1}\left|f(t+x)-g\left(t_{0}+x\right)\right| d x \\
& \quad \leq \int_{0}^{1}|f(t+x)-g(t+x)| d x \\
& \quad+\int_{0}^{1}\left|g(t+x)-g\left(t_{0}+x+\tau_{n}\right)\right| d x \\
& \quad+\int_{0}^{1}\left|g\left(t_{0}+x+\tau_{n}\right)-g\left(t_{0}+x\right)\right| d x \\
& \quad \forall t \in\left[t_{0}+\tau_{n}-\frac{l}{K_{0}}, t_{0}+\tau_{n}+\frac{l}{K_{0}}\right] .
\end{aligned}
$$

Since $f=g+h$, by (19), we have

$$
\begin{aligned}
\|h(t+x)\|_{L([0,1], R)} & =\int_{0}^{1}|h(t+x)| d x>\varepsilon \\
\forall t & \in\left[t_{0}+\tau_{n}-\frac{l}{K_{0}}, t_{0}+\tau_{n}+\frac{l}{K_{0}}\right] .
\end{aligned}
$$

Thus,

$$
\begin{aligned}
& \int_{t}^{t+1}|h(s)| d s>\varepsilon, \\
& \forall t \in\left[t_{0}+\tau_{n}-\frac{l}{K_{0}}, t_{0}+\tau_{n}+\frac{l}{K_{0}}\right],
\end{aligned}
$$

which contradicts

$$
\lim _{t \longrightarrow+\infty} \int_{t}^{t+1}|h(s)| d s=0 .
$$

Consequently, (15) holds. The proof is complete.

Definition 16. A function $g \in S(R \times X, R)$ if the following three conditions are true:

(i) for every $x \in X, g(\cdot, x) \in S(R, R)$,

(ii) the set $\{g(\cdot, x) \mid x \in X\}$ is uniformly bounded in the $S$-norm and equicontinuous in the $S$-norm,

(iii) for every $\varepsilon>0$, there exists a number $l>0$ such that every interval of length $l$ contains a number $\tau$ with the property

$$
\sup _{t \in R} \int_{t}^{t+1}|g(s+\tau, x)-g(s, x)| d s<\varepsilon, \quad \forall x \in X .
$$

Lemma 17. For a bounded continuous function $f: R \rightarrow X$, denote

$$
V_{f}(\tau)=\sup _{\theta \in R}\|f(\theta+\tau)-f(\theta)\|_{X}, \quad \tau \in R .
$$

Then $V_{f}$ satisfies the following properties:
(a) $V_{f}(\tau) \geq 0, V_{f}(-\tau)=V_{f}(\tau), \tau \in R$.
(b) $V_{f}(0)=0$.
(c) $V_{f}(t+s) \leq V_{f}(t)+V_{f}(s), s, t \in R$
(d) $f \in A P(R, X)$ if and only if $V_{f} \in A P(R, R)$.

Proof. Properties (a) through $(c)$ are easy to show. We only prove the property $(d)$. If $f \in A P(R, X)$, then for each $\varepsilon>0$ there exists $l>0$ such that in any interval of length $l$ of $R$ one can find a number $\tau \in(a, a+l)$ with the property

$$
V_{f}(\tau)=\sup _{\theta \in R}\|f(\theta+\tau)-f(\theta)\|_{X}<\varepsilon
$$

From $(c)$, we have $V_{f}(t+s)-V_{f}(t) \leq V_{f}(s)$ and $V_{f}(t)=$ $V_{f}(t+s-s) \leq V_{f}(t+s)+V_{f}(-s)$, then $-V_{f}(s)=-V_{f}(-s) \leq$ $V_{f}(t+s)-V_{f}(t), s, t \in R$. That is,

$$
\left|V_{f}(t+s)-V_{f}(t)\right| \leq V_{f}(s), \quad s, t \in R .
$$

Hence,

$$
\sup _{t \in R}\left|V_{f}(t+s)-V_{f}(t)\right| \leq V_{f}(s), \quad s, t \in R .
$$

It follows from (25) and (27) that

$$
\sup _{t \in R}\left|V_{f}(t+\tau)-V_{f}(t)\right| \leq V_{f}(\tau)<\varepsilon .
$$

Since $f \in A P(R, X)$, it is uniformly continuous. So, we know that, for every $\epsilon>0$, there exists $\delta>0$ such that if $|h|<\delta$ then $\|f(t+h)-f(t)\|_{X}<\epsilon, \forall t \in R$. Therefore, $V_{f}(h)<\epsilon$ and by (27) we get $\sup _{t \in R}\left|V_{f}(t+h)-V_{f}(t)\right|<\epsilon$, which implies that $V_{f}(t)$ is continuous. Thus, $V_{f}(t) \in A P(R, R)$. 
Conversely, if $V_{f} \in A P(R, R)$, then for each $\varepsilon>0$ there exists $l>0$ such that in any interval of length $l$ of $R$ one can find a number $\tau \in(a, a+l)$ with the property

$$
\left|V_{f}(t+\tau)-V_{f}(t)\right|<\varepsilon, \quad \forall t \in R .
$$

Hence, $V_{f}(\tau)=\sup _{\theta \in R}\|f(\theta+\tau)-f(\theta)\|_{X}<\varepsilon$. Since $V_{f}$ is continuous and $V_{f}(0)=0$, then for every $\epsilon>0$, there exists $\delta>0$ such that if $|h|<\delta$ we have $V_{f}(h)<\epsilon$. Therefore, $V_{f}(h)=\sup _{\theta \in R}\|f(\theta+h)-f(\theta)\|_{X}<\epsilon$, which implies $f$ is continuous. Thus, $f \in A P(R, X)$.

Lemma 18. Let $f \in \Lambda$, where $\Lambda$ consists of some almost periodic functions in Bohr sense from $R$ to $X, V(\tau)=$ $\sup _{f \in \Lambda} V_{f}(\tau)$ is finite. Then the family $\Lambda$ is uniformly almost periodic if and only if $V \in A P(R, R)$.

Proof. It is easy to find that $V$ satisfies the following properties:

(i) $V(t) \geq 0, V(-t)=V(t)$,

(ii) $V(0)=0$.

Moreover, by $(c)$ in Lemma 17 we have

$$
\begin{aligned}
V(t+s) & =\sup _{f \in \Lambda} V_{f}(t+s) \leq \sup _{f \in \Lambda}\left(V_{f}(t)+V_{f}(s)\right) \\
& \leq \sup _{f \in \Lambda} V_{f}(t)+\sup _{f \in \Lambda} V_{f}(s)=V(t)+V(s) .
\end{aligned}
$$

Similar to the proof of (27), we have

$$
\sup _{t \in R}|V(t+s)-V(t)| \leq V(s) .
$$

If $V \in A P(R, R)$, then for each $\varepsilon>0$, there exists $l>0$ such that in any interval of length $l$ of $R$ one can find a number $\tau \in(a, a+l)$ with the property

$$
\sup _{t \in R}|V(t+\tau)-V(t)|<\varepsilon .
$$

Noticing that $V(0)=0$, hence, $V(\tau)<\varepsilon$. Therefore, $V_{f}(\tau)<\varepsilon$ for all $f \in \Lambda$. So, the family $\Lambda$ is a uniformly almost periodic family.

Conversely, suppose that $\Lambda$ is a uniformly almost periodic family. Then for each $\varepsilon>0$ there exists $l>0$ such that in any interval of length $l$ of $R$ one can find a number $\tau \in(a, a+l)$ with the property

$$
\sup _{\theta \in R}\|f(\theta+\tau)-f(\theta)\|_{X}<\varepsilon, \quad \forall f \in \Lambda .
$$

From (33), we obtain that $V_{f}(\tau)<\varepsilon, \forall f \in \Lambda$ and $V(\tau) \leq \varepsilon$. By (31) we obtain that

$$
\sup _{t \in R}|V(t+\tau)-V(t)| \leq V(\tau) \leq \varepsilon .
$$

Besides, since $f \in \Lambda$ is continuous,

$$
V(h)=\sup _{f \in \Lambda} \sup _{\theta \in R}\|f(\theta+h)-f(\theta)\|_{X} \longrightarrow 0
$$

By (31) we obtain that $\sup _{t \in R}|V(t+h)-V(t)| \longrightarrow 0$ as $h \longrightarrow 0$, which implies that $V$ is continuous. Therefore, $V \in A P(R, R)$. The proof is completed.

Lemma 19. Let $\Lambda$ be a uniformly almost periodic family in Bohr sense. Then given a sequence $\left\{\alpha_{n}^{\prime}\right\}$, there exists a subsequence $\left\{\alpha_{n}\right\} \subset\left\{\alpha_{n}^{\prime}\right\}$ satisfying the following property: for every $\varepsilon>0$, there exists a constant $N$ such that

$$
\begin{aligned}
& \left\|f\left(t+\alpha_{n}\right)-f\left(t+\alpha_{m}\right)\right\|_{X}<\varepsilon, \\
& \qquad n, m>N \text {, for all } t \in R \text { and all } f \in \Lambda .
\end{aligned}
$$

Proof. According to Lemma 18, we know that $V(t)=$ $\sup _{f \in \Lambda} V_{f}(t)$ is almost periodic. By Lemma 3 we obtain that, for every sequence $\left\{\alpha_{n}^{\prime}\right\}$, there exists a subsequence $\left\{\alpha_{n}\right\}$ satisfying the following property: for each $\varepsilon>0$, there exists a constant $N>0$ such that

$$
\left|V\left(t+\alpha_{n}\right)-\mathrm{V}\left(t+\alpha_{m}\right)\right|<\varepsilon, \quad n, m>N, \forall t \in R .
$$

When $t=-\alpha_{m}$, we obtain $V\left(\alpha_{n}-\alpha_{m}\right)<\varepsilon$. Thus, $V_{f}\left(\alpha_{n}-\alpha_{m}\right)<$ $\varepsilon$ for all $f \in \Lambda$ and $n, m>N$. According to the definition of $V_{f}$, we have

$$
\begin{aligned}
& \left\|f\left(t+\alpha_{n}-\alpha_{m}\right)-f(t)\right\|_{X}<\varepsilon, \\
& \qquad n, m>N \text {, for all } t \in R \text { and } \forall f \in \Lambda .
\end{aligned}
$$

Hence, it is easy to see that

$$
\begin{aligned}
& \left\|f\left(t+\alpha_{n}\right)-f\left(t+\alpha_{m}\right)\right\|_{X}<\varepsilon, \\
& \qquad n, m>N \text {, for all } t \in R \text { and } \forall f \in \Lambda .
\end{aligned}
$$

The proof is completed.

Theorem 20. Let $g \in S(R \times X, R)$. Then for every sequence $\left\{\alpha_{n}^{\prime}\right\}$, there exists a subsequence $\left\{\alpha_{n}\right\} \subset\left\{\alpha_{n}^{\prime}\right\}$ such that $\{g(t+$ $\left.\left.\alpha_{n}, x\right), n \geq 1\right\}$ is convergent uniformly with respect to $x \in X$.

Proof. Since $g \in S(R \times X, R)$, we know that, for every $\varepsilon>0$, there exists a number $l>0$ such that every interval of length $l$ contains a number $\tau$ satisfying

$$
\sup _{t \in R} \int_{t}^{t+1}|g(s+\tau, x)-g(s, x)| d s<\varepsilon, \quad \forall x \in X .
$$

According to (40), we have

$$
\sup _{t \in R} \int_{0}^{1}|g(t+u+\tau, x)-g(t+u, x)| d u<\varepsilon,
$$

$\forall x \in X$.

Thus,

$$
\sup _{t \in R}\|g(t+u+\tau, x)-g(t+u, x)\|_{L([0,1], R)}<\varepsilon,
$$


From Lemma 8, we know that, for every fixed $x, g(t+$ $u, x) \in A P(R, L([0,1], R))$. Therefore, (42) implies that $\{g(t+$ $u, x), x \in X\}$ is a uniformly almost periodic family. From Lemma 19, we have that, for every sequence $\left\{\alpha_{n}^{\prime}\right\}$, there exists a subsequence $\left\{\alpha_{n}\right\} \subset\left\{\alpha_{n}^{\prime}\right\}$ such that, for every $\varepsilon>0$,

$$
\begin{array}{r}
\sup _{t \in R}\left\|g\left(t+u+\alpha_{n}, x\right)-g\left(t+u+\alpha_{m}, x\right)\right\|_{L([0,1], R)} \\
=\sup _{t \in R} \int_{t}^{t+1}\left|g\left(s+\alpha_{n}, x\right)-g\left(s+\alpha_{m}, x\right)\right| d s<\varepsilon, \\
n, m>N, \forall x \in X .
\end{array}
$$

Hence, $\left\{g\left(t+\alpha_{n}, x\right), n \geq 1\right\}$ is convergent uniformly with respect to $x \in X$.

Definition 21. A function $h \in M_{0}(R \times X, R)$ if the following two conditions are true:

(i) for every $x \in X, h(\cdot, x) \in M_{0}(R, R)$,

(ii) for every $\varepsilon>0$, there exists a constant $T>0$ such that

$$
\int_{t}^{t+1}|h(s, x)| d s<\varepsilon, \quad \forall t>T, \forall x \in X .
$$

Definition 22. A function $f \in A S(R \times X, R)$ if it can be expressed as

$$
f=g+h,
$$

where $g \in S(R \times X, R), h \in M_{0}(R \times X, R)$.

Theorem 23. The space of $A S(R, R)$ is a Banach space endowed with the norm

$$
\|f\|_{S}=\sup _{t \in R} \int_{t}^{t+1}|f(s)| d s .
$$

Proof. Let $\left\{f_{n}\right\} \subset A S(R, R)$ be a Cauchy sequence; i.e., for each $\varepsilon>0$, there exists a natural number $N>0$ such that

$$
\sup _{t \in R} \int_{t}^{t+1}\left|f_{n}(s)-f_{m}(s)\right| d s<\varepsilon, \quad n, m>N .
$$

Let $f_{n}=g_{n}+h_{n}, n \in N$, where $g_{n} \in S(R, R), h_{n} \in$ $M_{0}(R, R), n \in N$. By using Lemma 15 , we have

$$
\begin{aligned}
& \left\{g_{n}(t+x)-g_{m}(t+x), t \in R\right\} \\
& \quad \subset \overline{\left\{f_{n}(t+x)-f_{m}(t+x), t \in R\right\}} .
\end{aligned}
$$

Then, for every $\bar{\epsilon}>0$,

$$
\begin{aligned}
& \sup _{t \in R}\left\|g_{n}(t+x)-g_{m}(t+x)\right\|_{L([0,1], R)} \\
& \quad<\sup _{t \in R}\left\|f_{n}(t+x)-f_{m}(t+x)\right\|_{L([0,1], R)}+\bar{\epsilon} .
\end{aligned}
$$

According to the arbitrariness of $\bar{\epsilon}$, we obtain

$$
\begin{aligned}
\sup _{t \in R} & \left\|g_{n}(t+x)-g_{m}(t+x)\right\|_{L([0,1], R)} \\
& \leq \sup _{t \in R}\left\|f_{n}(t+x)-f_{m}(t+x)\right\|_{L([0,1], R)} .
\end{aligned}
$$

Thus,

$$
\begin{aligned}
& \sup _{t \in R} \int_{t}^{t+1}\left|g_{n}(s)-g_{m}(s)\right| d s \\
& \quad=\sup _{t \in R} \int_{0}^{1}\left|g_{n}(x+t)-g_{m}(x+t)\right| d x \\
& \quad \leq \sup _{t \in R} \int_{0}^{1}\left|f_{n}(t+x)-f_{m}(t+x)\right| d x \\
& \quad=\sup _{t \in R} \int_{t}^{t+1}\left|f_{n}(s)-f_{m}(s)\right| d s<\varepsilon, \quad n, m>N,
\end{aligned}
$$

which means that $\left\{g_{n}\right\} \subset S(R, R)$ is a Cauchy sequence. So $\lim _{n \rightarrow+\infty} g_{n}=g \in S(R, R)$. Similarly, we can obtain

$$
\begin{aligned}
& \sup _{t \in R} \int_{t}^{t+1}\left|h_{n}(s)-h_{m}(s)\right| d s \\
& \leq \sup _{t \in R} \int_{t}^{t+1}\left|f_{n}(s)-f_{m}(s)\right| d s \\
& \quad+\sup _{t \in R} \int_{t}^{t+1}\left|g_{n}(s)-g_{m}(s)\right| d s \leq 2 \varepsilon,
\end{aligned}
$$

$$
n, m>N
$$

According to Lemma 14, we obtain $\lim _{n \rightarrow+\infty} h_{n}=h \in$ $M_{0}(R, R)$ and $\lim _{n \rightarrow+\infty} f_{n}=f=g+h \in A S(R, R)$. The proof is completed.

\section{Stepanov-Like Asymptotical Almost Periodic Solutions of Semilinear Delay Differential Equations}

In this section, we investigate the existence and uniqueness of Stepanov-like asymptotical almost periodic solutions for the following semilinear differential equation:

$$
x^{\prime}(t)+A(t) x(t)=f(t, x(t), x(t-\tau)), \quad t \in R,
$$

where $A \in A P(R, R), f \in A S(R \times R \times R, R)$, and $\tau>0$ is a constant.

We make some assumption:

$\left(H_{1}\right) \quad A \in A P(R, R)$ and $A^{*}=\inf _{t \in R} A(t)>0$.

$\left(H_{2}\right) f=f_{1}+f_{2} \in A S(R \times R \times R, R)$, where $f_{1} \in S(R \times R \times$ $R, R), f_{2} \in M_{0}(R \times R \times R, R)$.

$\left(H_{3}\right)$ There exist constants $L_{1}, L_{2}>0$ such that, for all $t \in R$ and for all $u_{1}, u_{2}, v_{1}, v_{2} \in R$,

$$
\begin{gathered}
\left|f_{1}\left(t, u_{1}, v_{1}\right)-f_{1}\left(t, u_{2}, v_{2}\right)\right| \\
\quad \leq L_{1}\left(\left|u_{1}-u_{2}\right|+\left|v_{1}-v_{2}\right|\right), \\
\left|f_{2}\left(t, u_{1}, v_{1}\right)-f_{2}\left(t, u_{2}, v_{2}\right)\right| \\
\quad \leq L_{2}\left(\left|u_{1}-u_{2}\right|+\left|v_{1}-v_{2}\right|\right) .
\end{gathered}
$$


Lemma 24. Let $x \in A S(R, R)$ and $\left(H_{2}\right),\left(H_{3}\right)$ hold; then $f(\cdot, x(\cdot), x(\cdot-\tau)) \in A S(R, R)$.

Proof. Since $x \in A S(R, R)$, we have $x=x_{1}+x_{2}$, where $x_{1} \in$ $S(R, R), x_{2} \in M_{0}(R, R)$. Then function $f(t, x(t), x(t-\tau))$ can be written in the form:

$$
\begin{aligned}
f(t, x(t), x(t-\tau))= & f_{1}\left(t, x_{1}(t), x_{1}(t-\tau)\right) \\
& +f(t, x(t), x(t-\tau)) \\
& -f\left(t, x_{1}(t), x_{1}(t-\tau)\right) \\
& +f\left(t, x_{1}(t), x_{1}(t-\tau)\right) \\
& -f_{1}\left(t, x_{1}(t), x_{1}(t-\tau)\right) .
\end{aligned}
$$

Step 1. We prove $f_{1}\left(\cdot, x_{1}(\cdot), x_{1}(\cdot-\tau)\right) \in S(R, R)$. Let $\varepsilon>0$. By Lemma 9 , for every sequence $\left\{h_{k} ; k \geq 1\right\} \subset R$, there exists a subsequence $\left\{h_{1 k} ; k \geq 1\right\} \subset\left\{h_{k} ; k \geq 1\right\}$ such that the sequence $\left\{x_{1}\left(t+h_{1 k}\right) ; k \geq 1\right\}$ is convergent. From Theorem 20, it follows that $\left\{f_{1}\left(t+h_{1 k}, u, v\right) ; k \geq 1\right\}$ is also convergent uniformly with respect to $u, v \in R$. Therefore, for any $\varepsilon>0$, there exist positive integers $N_{1}$ and $N_{2}$ such that

$$
\begin{aligned}
& \sup _{t \in R} \int_{t}^{t+1}\left|x_{1}\left(s+h_{1 k}\right)-x_{1}\left(s+h_{1 p}\right)\right| d s<\frac{\varepsilon}{4 L_{1}}, \\
& k, p \geq N_{1}
\end{aligned}
$$

and

$$
\begin{array}{r}
\sup _{t \in R} \int_{t}^{t+1}\left|f_{1}\left(s+h_{1 k}, u, v\right)-f_{1}\left(s+h_{1 p}, u, v\right)\right| d s<\frac{\varepsilon}{2}, \\
k, p \geq N_{2}, \forall u, v \in R .
\end{array}
$$

Hence

$$
\begin{aligned}
& \sup _{t \in R} \int_{t}^{t+1} \mid f_{1}\left(s+h_{1 k}, x_{1}\left(s+h_{1 k}\right), x_{1}\left(s+h_{1 k}-\tau\right)\right) \\
& \quad-f_{1}\left(s+h_{1 p}, x_{1}\left(s+h_{1 p}\right), x_{1}\left(s+h_{1 p}-\tau\right)\right) \mid d s \\
& \quad \leq \sup _{t \in R} \int_{t}^{t+1} \mid f_{1}(s \\
& \left.\quad+h_{1 k}, x_{1}\left(s+h_{1 k}\right), x_{1}\left(s+h_{1 k}-\tau\right)\right)-f_{1}(s \\
& \left.\quad+h_{1 k}, x_{1}\left(s+h_{1 p}\right), x_{1}\left(s+h_{1 p}-\tau\right)\right) \mid d s \\
& \quad+\sup _{t \in R}^{t+1} \mid f_{1}(s \\
& \left.\quad+h_{1 k}, x_{1}\left(s+h_{1 p}\right), x_{1}\left(s+h_{1 p}-\tau\right)\right)-f_{1}(s
\end{aligned}
$$

$$
\begin{aligned}
& \left.+h_{1 p}, x_{1}\left(s+h_{1 p}\right), x_{1}\left(s+h_{1 p}-\tau\right)\right) \mid d s \\
& \leq L_{1} \sup _{t \in R} \int_{t}^{t+1}\left(\left|x_{1}\left(s+h_{1 k}\right)-x_{1}\left(s+h_{1 p}\right)\right|\right. \\
& \left.+\left|x_{1}\left(s+h_{1 k}-\tau\right)-x_{1}\left(s+h_{1 p}-\tau\right)\right|\right) d s \\
& +\frac{\varepsilon}{2} \leq L_{1} \frac{\varepsilon}{2 L_{1}}+\frac{\varepsilon}{2}=\varepsilon, \\
& \quad \text { for } k, p \geq N=\max \left\{N_{1}, N_{2}\right\} .
\end{aligned}
$$

Therefore, $f_{1}\left(\cdot, x_{1}(\cdot), x_{1}(\cdot-\tau)\right) \in S(R, R)$.

Step 2. We prove that

$$
\begin{aligned}
& f(t, x(t), x(t-\tau))-f\left(t, x_{1}(t), x_{1}(t-\tau)\right) \\
& \quad \in M_{0}(R, R)
\end{aligned}
$$

and

$$
\begin{aligned}
& f\left(t, x_{1}(t), x_{1}(t-\tau)\right)-f_{1}\left(t, x_{1}(t), x_{1}(t-\tau)\right) \\
& \quad \in M_{0}(R, R) .
\end{aligned}
$$

From $\left(H_{3}\right)$, we have

$$
\begin{aligned}
& \lim _{t \rightarrow \infty} \int_{t}^{t+1} \mid f(s, x(s), x(s-\tau)) \\
& \quad-f\left(s, x_{1}(s), x_{1}(s-\tau)\right) \mid d s \leq \lim _{t \rightarrow \infty} \int_{t}^{t+1}\left(L_{1}\right. \\
& \left.\quad+L_{2}\right)\left(\left|x_{2}(s)\right|+\left|x_{2}(s-\tau)\right|\right) d s=0 .
\end{aligned}
$$

According to the definition of $f$ and Definition 21, we have

$$
\begin{aligned}
& f\left(t, x_{1}(t), x_{1}(t-\tau)\right)-f_{1}\left(t, x_{1}(t), x_{1}(t-\tau)\right) \\
& \quad=f_{2}\left(t, x_{1}(t), x_{1}(t-\tau)\right)
\end{aligned}
$$

and

$$
\lim _{t \rightarrow \infty} \int_{t}^{t+1}\left|f_{2}\left(s, x_{1}(s), x_{1}(s-\tau)\right)\right| d s=0 .
$$

Hence, $f(\cdot, x(\cdot), x(\cdot-\tau)) \in A S(R, R)$. The proof is complete.

Theorem 25. Assume $\left(H_{1}\right)-\left(H_{3}\right)$ hold. If $\Delta<1$, where $\Delta=$ $4\left(L / A^{*}\right), L=\max \left\{L_{1}, L_{2}\right\}$, then system (53) has a unique Stepanov-like asymptotical almost periodic solution.

Proof. For any $\varphi \in A S(R, R)$, consider the linear differential equation

$$
x^{\prime}(t)+A(t) x(t)=f(t, \varphi(t), \varphi(t-\tau)) .
$$

Since $\left(H_{1}\right)$ holds, by the exponential dichotomy of linear differential equation, (64) has a unique bounded solution

$$
x^{\varphi}(t)=\int_{-\infty}^{t} e^{-\int_{s}^{t} A(u) d u} f(s, \varphi(s), \varphi(s-\tau)) d s,
$$


Define an operator $F: A S(R, R) \longrightarrow M(R, R)$ by setting $F \varphi=$ $x^{\varphi}$ for every $\varphi \in A S(R, R)$.

Step 1 ( $F$ is self-mapping). From Lemma 24 , we have $\widetilde{f}(\cdot)=$ $f(\cdot, \varphi(t), \varphi(\cdot-\tau)) \in A S(R, R)$. Let $\widetilde{f}=\widetilde{f_{1}}+\widetilde{f_{2}}$, where $\widetilde{f_{1}} \in$ $S(R, R), \widetilde{f_{2}} \in M_{0}(R, R)$, and define

$$
\begin{aligned}
& F_{1}(t)=\int_{-\infty}^{t} e^{-\int_{s}^{t} A(u) d u} \widetilde{f_{1}}(s) d s, \\
& F_{2}(t)=\int_{-\infty}^{t} e^{-\int_{s}^{t} A(u) d u} \widetilde{f_{2}}(s) d s .
\end{aligned}
$$

By Lemmas 8 and 10, there exists $l>0$ such that every interval of length $l$ contains a number $\theta$ such that

$$
\begin{array}{r}
|A(t+\theta)-A(t)|<\varepsilon, \\
\sup _{t \in R} \int_{t}^{t+1}\left|\widetilde{f}_{1}(s+\theta)-\widetilde{f}_{1}(s)\right| d s<\varepsilon .
\end{array}
$$

Then

$$
\begin{aligned}
& \left\|F_{1}(t+\theta)-F_{1}(t)\right\|_{S}=\sup _{t \in R} \int_{t}^{t+1} \mid \int_{-\infty}^{s+\theta} e^{-\int_{w}^{s+\theta} A(u) d u} \widetilde{f_{1}}(w) d w \\
& -\int_{-\infty}^{s} e^{-\int_{w}^{s} A(u) d u} \widetilde{f}_{1}(w) d w \mid d s \\
& =\sup _{t \in R} \int_{t}^{t+1} \mid \int_{-\infty}^{s} e^{-\int_{w}^{s} A(u+\theta) d u} \widetilde{f}_{1}(w+\theta) d w \\
& -\int_{-\infty}^{s} e^{-\int_{w}^{s} A(u) d u} \widetilde{f}_{1}(w) d w \mid d s \\
& \leq \sup _{t \in R} \int_{t}^{t+1} \mid \int_{-\infty}^{s} e^{-\int_{w}^{s} A(u+\theta) d u} \widetilde{f_{1}}(w+\theta) d w \\
& -\int_{-\infty}^{s} e^{-\int_{w}^{s} A(u) d u} \widetilde{f_{1}}(w+\theta) d w \mid d s \\
& +\sup _{t \in R} \int_{t}^{t+1} \mid \int_{-\infty}^{s} e^{-\int_{w}^{s} A(u) d u} \widetilde{f}_{1}(w+\theta) d w \\
& -\int_{-\infty}^{s} e^{-\int_{w}^{s} A(u) d u} \widetilde{f}_{1}(w) d w \mid d s \\
& =\sup _{t \in R} \int_{t}^{t+1} \mid \int_{-\infty}^{\mathcal{s}}\left(e^{-\int_{w}^{s} A(u+\theta) d u}-e^{-\int_{w}^{s} A(u) d u}\right) \\
& \cdot \widetilde{f}_{1}(w+\theta) d w \mid d s \\
& +\sup _{t \in R} \int_{t}^{t+1} \int_{-\infty}^{s} e^{-\int_{w}^{s} A(u) d u}\left|\widetilde{f}_{1}(w+\theta)-\widetilde{f}_{1}(w)\right| d w d s \\
& \leq \sup _{t \in R} \int_{t}^{t+1} \mid \int_{-\infty}^{s} e^{-A^{*}(s-w)}\left(\int_{w}^{s}|A(u+\theta)-A(u)| d u\right) \\
& \cdot \widetilde{f}_{1}(w+\theta) d w \mid d s
\end{aligned}
$$

$$
\begin{aligned}
& +\sup _{t \in R} \int_{t}^{t+1} \int_{-\infty}^{s} e^{-\int_{w}^{s} A(u) d u}\left|\widetilde{f}_{1}(w+\theta)-\widetilde{f}_{1}(w)\right| d w d s \\
& \leq \varepsilon \sup _{t \in R} \int_{t}^{t+1} \int_{0}^{+\infty} e^{-A^{*} \sigma} \sigma\left|\widetilde{f}_{1}(s-\sigma+\theta)\right| d \sigma d s \\
& +\int_{0}^{+\infty} e^{-A^{*} \sigma} \sup _{t \in R} \int_{t}^{t+1}\left|\widetilde{f}_{1}(s-\sigma+\theta)-\widetilde{f}_{1}(s-\sigma)\right| d s d \sigma \\
& \leq\left\|\widetilde{f_{1}}\right\|_{S} \varepsilon \frac{1}{\left(A^{*}\right)^{2}}+\varepsilon \frac{1}{A^{*}},
\end{aligned}
$$

where $\sigma=s-w$. Hence, $F_{1} \in S(R, R)$.

Since $\widetilde{f_{2}} \in M_{0}(R, R)$, we have

$$
\begin{aligned}
& \lim _{t \rightarrow+\infty} \int_{t}^{t+1}\left|F_{2}(s)\right| d s \\
& \quad=\lim _{t \rightarrow+\infty} \int_{t}^{t+1}\left|\int_{-\infty}^{s} e^{-\int_{w}^{s} A(u) d u} \widetilde{f_{2}}(w) d w\right| d s \\
& \quad \leq \lim _{t \rightarrow+\infty} \int_{t}^{t+1} \int_{-\infty}^{s} e^{-A^{*}(s-w)}\left|\widetilde{f_{2}}(w)\right| d w d s \\
& \quad=\lim _{t \rightarrow+\infty} \int_{t}^{t+1} \int_{0}^{+\infty} e^{-A^{*} \xi}\left|\widetilde{f_{2}}(s-\xi)\right| d \xi d s \\
& \quad=\lim _{t \rightarrow+\infty} \int_{0}^{+\infty} e^{-A^{*} \xi} \int_{t}^{t+1}\left|\widetilde{f}_{2}(s-\xi)\right| d s d \xi
\end{aligned}
$$

where $\xi=s-w$. According to Lebesgue's dominated convergence theorem, we obtain $F_{2} \in M_{0}(R, R)$. Therefore, $F$ is self-mapping.

Step 2 ( $F$ is a contraction mapping). $\forall \varphi, \psi \in A S(R, R)$, we have

$$
\begin{aligned}
& \|F \varphi-F \psi\|_{S} \\
& \quad=\sup _{t \in R} \int_{t}^{t+1} \mid \int_{-\infty}^{s} e^{-\int_{w}^{s} A(u) d u} f(w, \varphi(w), \varphi(w-\tau)) d w \\
& \quad-\int_{-\infty}^{s} e^{-\int_{w}^{s} A(u) d u} f(w, \psi(w), \psi(w-\tau)) d w \mid d s \\
& \quad \leq \sup _{t \in R} \int_{t}^{t+1} \int_{-\infty}^{s} e^{-A^{*}(s-w)} 2 L(|\varphi(w)-\psi(w)| \\
& +|\varphi(w-\tau)-\psi(w-\tau)|) d w d s \\
& =2 L \sup _{t \in R} \int_{t}^{t+1} \int_{0}^{+\infty} e^{-A^{*} \sigma}(|\varphi(s-\sigma)-\psi(s-\sigma)| \\
& +|\varphi(s-\sigma-\tau)-\psi(s-\sigma-\tau)|) d \sigma d s \\
& \quad \leq 2 L \int_{0}^{+\infty} e^{-A^{*} \sigma} 2\|\varphi-\psi\|_{S} d u=\frac{4 L}{A^{*}}\|\varphi-\psi\|_{S} .
\end{aligned}
$$

Hence, $T$ has a unique fixed point in $A S(R, R)$. Therefore, system (53) has a unique Stepanov-like asymptotical almost periodic solution. The proof is complete. 
An example: consider the following equation:

$$
\begin{aligned}
x^{\prime}(t) & +(\sin t+5) x(t) \\
= & \frac{1}{2}(\sin x(t)+\sin x(t-\tau)) \\
& +\frac{1}{2} e^{-|t|}(\cos x(t)+\cos x(t-\tau)),
\end{aligned}
$$

where $\tau$ is a positive constant. In this case, $A(t)=\sin t+5$,

$$
\begin{aligned}
& f(t, x(t), x(t-\tau)) \\
& =\frac{1}{2}(\sin x(t)+\sin x(t-\tau)) \\
& +\frac{1}{2} e^{-|t|}(\cos x(t)+\cos x(t-\tau)), \\
& f_{1}(t, x(t), x(t-\tau))=\frac{1}{2}(\sin x(t)+\sin x(t-\tau)), \\
& f_{2}(t, x(t), x(t-\tau)) \\
& =\frac{1}{2} e^{-|t|}(\cos x(t)+\cos x(t-\tau)) .
\end{aligned}
$$

Obviously, $A^{*}=4$,

$$
\begin{aligned}
& \left|f_{1}(t, x(t), x(t-\tau))-f_{1}(t, y(t), y(t-\tau))\right| \\
& \quad \leq \frac{1}{2}(|x(t)-y(t)|+|x(t-\tau)-y(t-\tau)|), \\
& \left|f_{2}(t, x(t), x(t-\tau))-f_{2}(t, y(t), y(t-\tau))\right| \\
& \quad \leq \frac{1}{2}(|x(t)-y(t)|+|x(t-\tau)-y(t-\tau)|) .
\end{aligned}
$$

Hence, $4 L / A^{*}=1 / 2<1$. Thus, all the conditions of Theorem 25 are satisfied. By Theorem 25, system (71) has a unique Stepanov-like asymptotical almost periodic solution.

\section{Data Availability}

No data were used to support this study.

\section{Conflicts of Interest}

The authors declare that they have no conflicts of interest.

\section{Acknowledgments}

This work is supported by the National Natural Sciences Foundation of China under Grants No. 11861072 and No. 11361072 .

\section{References}

[1] H. Bohr, "Zur theorie der fast periodischen funktionen," Acta Mathematica, vol. 45, no. 1, pp. 29-127, 1925.

[2] H. Bohr, "Zur Theorie der Fastperiodischen FUNktionen," Acta Mathematica, vol. 46, no. 1-2, pp. 101-214, 1925.
[3] A. S. Besicovitch, Almost Periodic Functions, Dover, New York, NY, USA, 1954.

[4] B. Amir and L. Maniar, "Composition of pseudo-almost periodic functions and Cauchy problems with operator of nondense domain," Annales MathÉmatiques Blaise Pascal, vol. 6, no. 1, pp. 1-11, 1999.

[5] L. Amerio and G. Prouse, Almost-periodic functions and functional equations, Van Nostrand Reinhold Co., New YorkToronto, Ont.-Melbourne, 1971.

[6] J. Andres and D. Pennequin, "On Stepanov almost-periodic oscillations and their discretizations," Journal of Difference Equations and Applications, vol. 18, no. 10, pp. 1665-1682, 2012.

[7] J. Andres and D. Pennequin, "On the nonexistence of purely Stepanov almost-periodic solutions of ordinary differential equations," Proceedings of the American Mathematical Society, vol. 140, no. 8, pp. 2825-2834, 2012.

[8] Z. Hu, "Boundedness and Stepanov's almost periodicity of solutions," Electronic Journal of Differential Equations, No. 35, 7 pages, 2005.

[9] Z. Hu and A. B. Mingarelli, "Bochner's theorem and Stepanov almost periodic functions," Annali di Matematica Pura ed Applicata. Series IV, vol. 187, no. 4, pp. 719-736, 2008.

[10] M. Maqbul and D. Bahuguna, "Almost periodic solutions for Stepanov-almost periodic differential equations," Differential Equations and Dynamical Systems, vol. 22, no. 3, pp. 251-264, 2014.

[11] Y. Li and P. Wang, "Almost periodic solution for neutral functional dynamic equations with Stepanov-almost periodic terms on time scales," Discrete and Continuous Dynamical Systems Series S, vol. 10, no. 3, pp. 463-473, 2017.

[12] T. Diagana, "Stepanov-like pseudo almost periodic functions and their applications to differential equations," Communications in Mathematical Analysis, vol. 3, no. 1, pp. 9-18, 2007.

[13] C. Y. Zhang, "Pseudo-almost-periodic solutions of some differential equations," Journal of Mathematical Analysis and Applications, vol. 181, no. 1, pp. 62-76, 1994.

[14] T. Diagana, G. M. Mophou, and G. M. N'Guerekata, "Existence of weighted pseudo almost periodic solutions to some classes of differential equations with Sp-weighted pseudo almost periodic coefficients," Nonlinear Analysis, vol. 72, no. 1, pp. 430-438, 2010.

[15] T. Diagana and G. M. N'Guerekata, "Stepanov-like almost automorphic functions and applications to some semilinear equations," Applicable Analysis: An International Journal, vol. 86, no. 6, pp. 723-733, 2007.

[16] T. Diagana, "Existence of pseudo almost automorphic solutions to some abstract differential equations with Sp-pseudo almost automorphic coefficients," Nonlinear Analysis, vol. 70, no. 11, pp. 3781-3790, 2009.

[17] M. Frechet, Les fonctions asymptotiquement presque-periodiques continues, vol. 213, pp. 520-522, C. R. National Academy of Sciences, Paris, 1941.

[18] M. Frechet, "Les fonctions asymptotiquement presqueperiodiques," Revue Science, vol. 79, pp. 341-354, 1941.

[19] T. Diagana, Almost Automorphic Type and Almost Periodic Type Functions in Abstract Spaces, Springer, Berlin, Germany, 2013.

[20] B. de Andrade and C. Lizama, "Existence of asymptotically almost periodic solutions for damped wave equations," Journal of Mathematical Analysis and Applications, vol. 382, no. 2, pp. 761-771, 2011. 
[21] J. Cao, A. Debbouche, and Y. Zhou, "Asymptotically almost periodicity for a class of Weyl-Liouville fractional evolution equations," Mediterranean Journal of Mathematics, vol. 15, no. 4, Art. 155, 22 pages, 2018.

[22] J. Cao, Q. Yang, Z. Huang, and Q. Liu, "Asymptotically almost periodic solutions of stochastic functional differential equations," Applied Mathematics and Computation, vol. 218, no. 5, pp. 1499-1511, 2011.

[23] Y. Tang and S. Xie, "Global attractivity of asymptotically almost periodic Nicholson's blowflies models with a nonlinear densitydependent mortality term," International Journal of Biomathematics, vol. 11, no. 6, 1850079, 15 pages, 2018.

[24] C. Huang, B. Liu, X. Tian, L. Yang, and X. Zhang, "Global Convergence on Asymptotically Almost Periodic SICNNs with Nonlinear Decay Functions," Neural Processing Letters, 2018.

[25] L. Yao, "Dynamics of Nicholson's blowflies models with a nonlinear density-dependent mortality," Applied Mathematical Modelling: Simulation and Computation for Engineering and Environmental Systems, vol. 64, pp. 185-195, 2018.

[26] D. N. Cheban, Asymptotically Almost Periodic Solutions of Differential Equations, Hindawi Publishing Corporation, New York, NY, USA, 2009.

[27] C. Corduneanu, Almost Periodic Oscillations and Waves, Springer, New York, NY, USA, 2009.

[28] A. M. Fink, Almost Periodic Differential Equations, SpringerVerlag, Berlin, Germany, 1974.

[29] B. M. Levitan and V. V. Zhikov, Almost-periodic functions and functional differential equations, Cambridge University Press, Cambridge, 1982. 


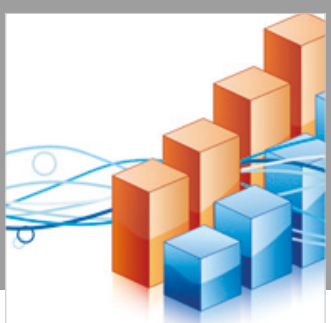

Advances in

Operations Research

\section{-n-m}
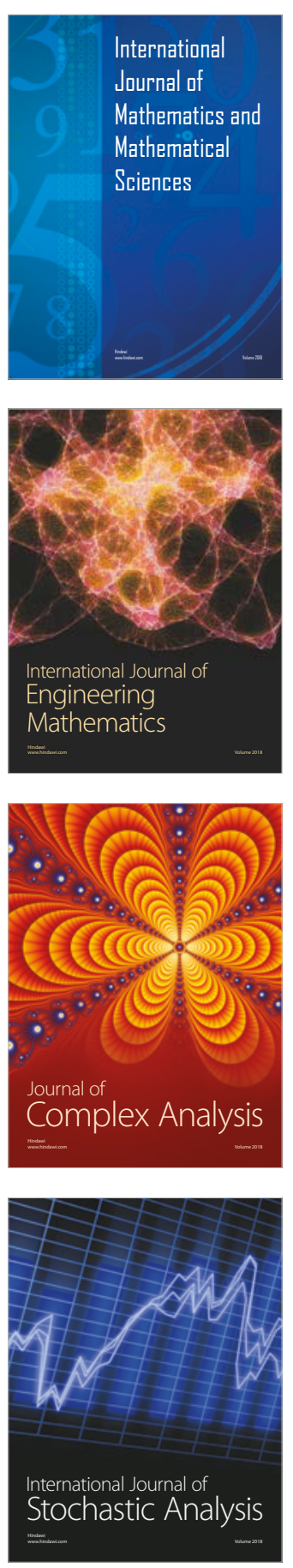
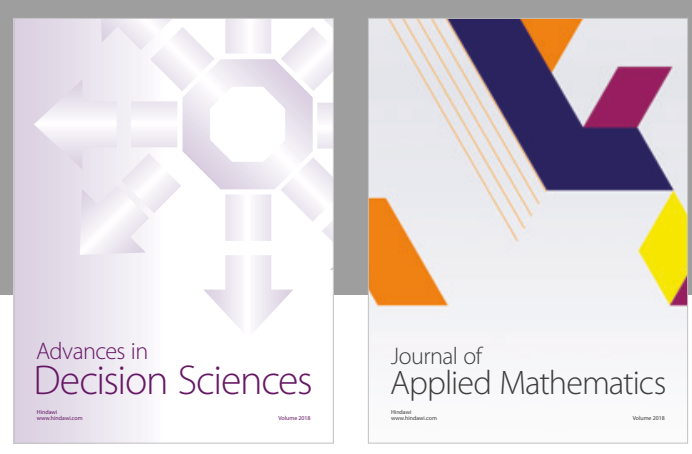

Journal of

Applied Mathematics
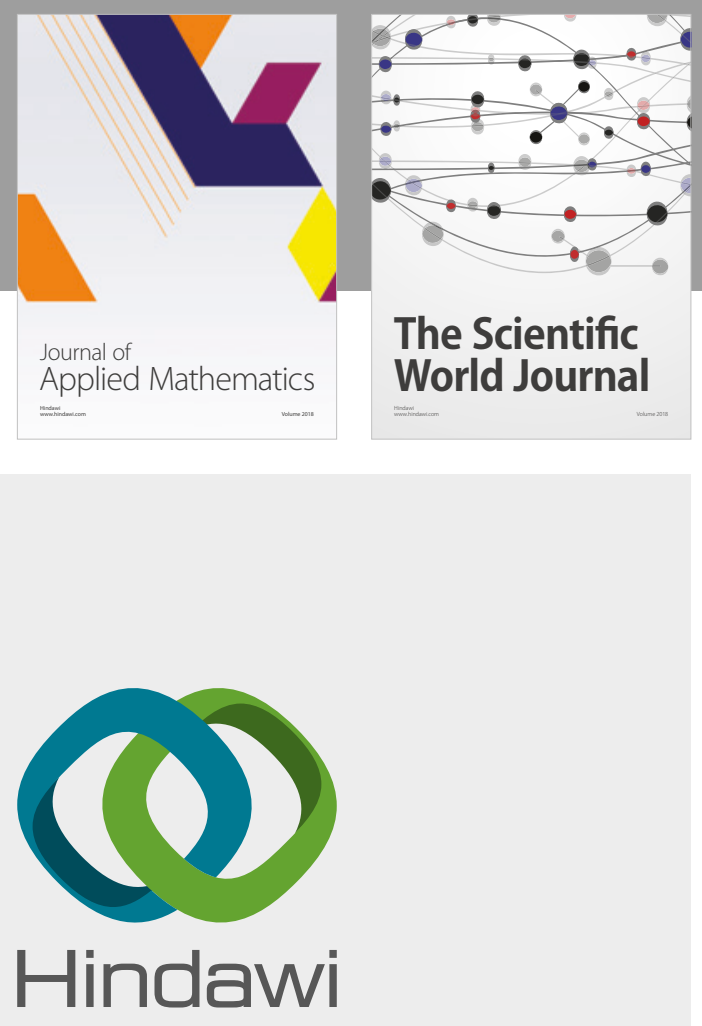

Submit your manuscripts at

www.hindawi.com

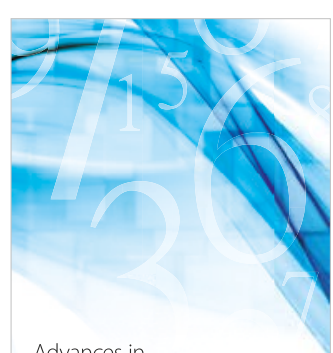

Advances in
Numerical Analysis
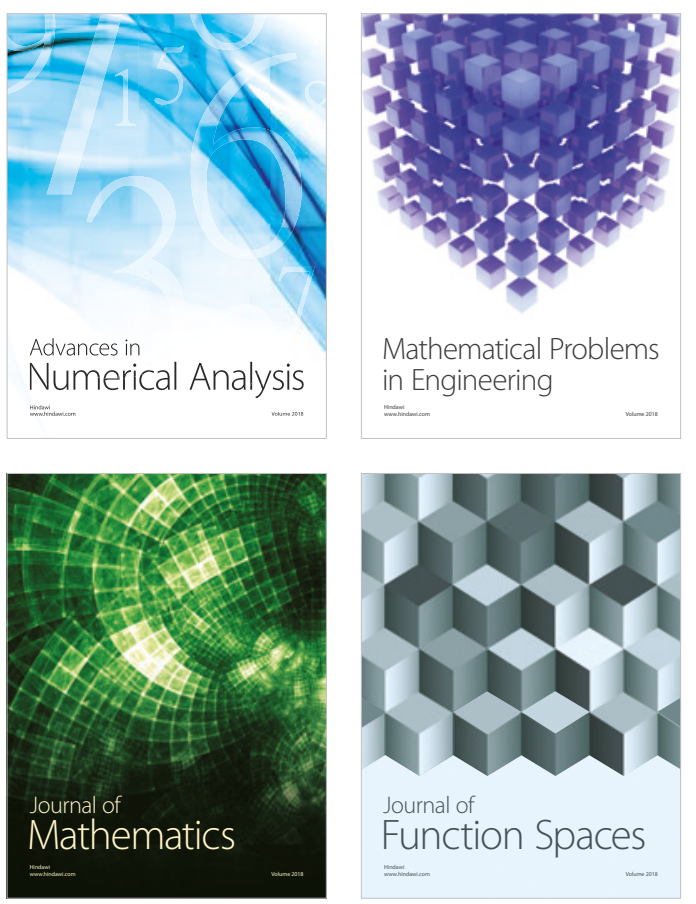

Mathematical Problems in Engineering

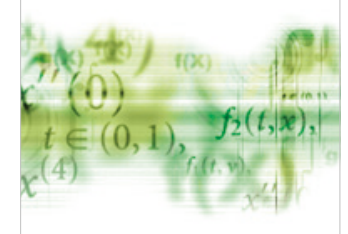

International Journal of

Differential Equations

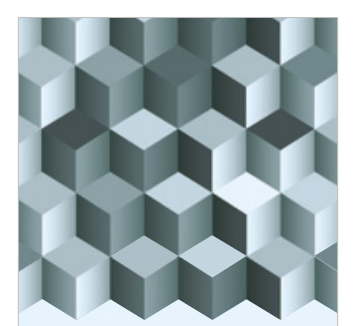

Journal of

Function Spaces

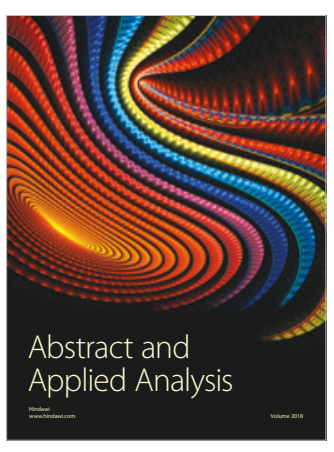

The Scientific

World Journal

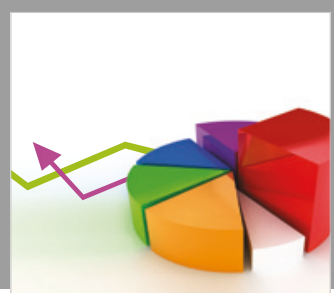

Journal of

Probability and Statistics
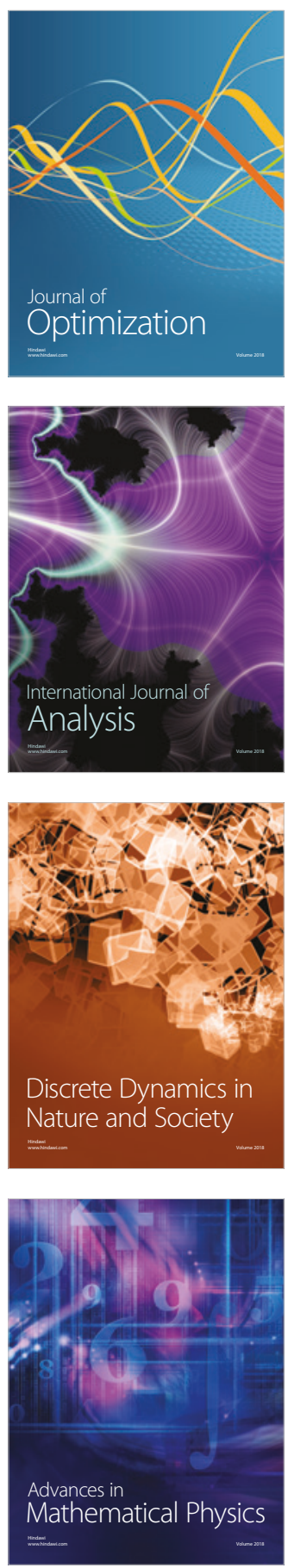